\title{
The NOTCH1-HEY1 pathway regulates self-renewal and epithelial-mesenchymal transition of salivary adenoid cystic carcinoma cells
}

\author{
Jing Xie ${ }^{1,3,4^{*}}$, Li-song Lin²* Xiao-yu Huang1,3,4*, Rui-huan Gan ${ }^{1,3}$, Lin-can Ding1, Bo-hua Su1, Yong Zhao ${ }^{3,5}$, \\ You-guang Lu ${ }^{1,3 凶}$, Da-li Zheng ${ }^{3 凶}$ \\ 1. Department of Preventive Dentistry, School and Hospital of Stomatology, Fujian Medical University, 246 Yang Qiao Middle Road, Fuzhou 350000 , China \\ 2. Department of Oral and Maxillofacial Surgery, Affiliated First Hospital of Fujian Medical University, 20 Cha Zhong Road, Fuzhou 350005, China \\ 3. Key laboratory of Stomatology of Fujian Province, School and Hospital of Stomatology, Fujian Medical University, 88 Jiaotong Rd, Fuzhou 350004, China \\ 4. Key Laboratory of Ministry of Education for Gastrointestinal Cancer, Fujian Medical University, 1 Xue Yuan Road, University Town, Fuzhou 350122, China \\ 5. Department of pathology, School and Hospital of Stomatology, Fujian Medical University, 246 Yang Qiao Middle Road, Fuzhou 350000, China \\ * These authors contributed equally to this paper \\ $\triangle$ Corresponding authors: Da-Li Zheng. Ph.D, Email: dalizheng@fjmu.edu.cn; Phone: +86-591-83720599; Fax number: +86-591-83720599; Postal address: Key \\ laboratory of Stomatology of Fujian Province, School and Hospital of Stomatology, Fujian Medical University, 88 Jiao Tong Road, Fuzhou 350004, China or \\ You-Guang Lu. Ph.D, Email: fjlyg63@fjmu.edu.cn; Phone: +86-591-83736429; Fax number: +86-591-83720599; Postal address: Department of Preventive Dentistry, \\ School and Hospital of Stomatology, Fujian Medical University, 246 Yang Qiao Middle Road, Fuzhou 350000, China.
}

( ) The author(s). This is an open access article distributed under the terms of the Creative Commons Attribution License (https://creativecommons.org/licenses/by/4.0/). See http://ivyspring.com/terms for full terms and conditions.

Received: 2019.05.06; Accepted: 2019.11.12; Published: 2020.01.01

\begin{abstract}
Our previous study demonstrated a close relationship between the NOTCH signaling pathway and salivary adenoid cystic carcinoma (SACC). Its receptor gene, NOTCHI, and its downstream gene, HESI, contribute to the proliferation, invasion and metastasis of SACC. Accumulating evidence supports HEY 1 as another effector of the signaling pathway. The purpose of this study was to explore the effects of the NOTCHI-HEYI pathway on the proliferation, invasion and metastasis of SACC cells. Our results verified that $\mathrm{HEY} 1$ is a specific molecular target of the NOTCH signaling pathway in SACC cells and that its expression in carcinoma is much higher than that in paracarcinoma tissues. The expression of $\mathrm{NOTCH} \mathrm{l}$ and $\mathrm{HEYI}$ are positively correlated in the salivary adenoid cystic carcinoma tissues. NOTCHI is significantly related to the activation of $\mathrm{HEY} 1$ in $\mathrm{SACC}$, and that $\mathrm{HEY} 1$ reciprocally regulates $\mathrm{NOTCH} 1$ expression in SACC. HEYl promotes cell proliferation and spheroid formation and inhibits cell apoptosis in vitro. In addition, HEYl enhances the tumorigenicity of SACC in vivo. Furthermore, HEYl increases cell invasion and metastasis by driving the expression of epithelial-mesenchymal transition (EMT)-related genes and MMPs. The results of this study indicate that the NOTCHI-HEYl pathway is specifically upregulated in SACC and promotes cell proliferation, self-renewal, invasion, metastasis and the expression of EMT-related genes and MMPs. Our findings suggest that a NOTCHI-HEYl pathway inhibitor might therefore have potential therapeutic applications in treating SACC patients by inhibiting cancer cell growth and metastasis.
\end{abstract}

Key words: SACC, NOTCH1, HEY1, cancer stem cells, proliferation, invasion, EMT

\section{Introduction}

SACC is an aggressive tumor with unpredictable and unique biological destruction that exhibits features such as slow but relentless growth, a propensity for peripheral nerve and blood vessel invasion, a high incidence of distant lung metastasis and a high risk of relapse [1-4]. As a result, the survival rates after a diagnosis of SACC are $35 \%$ at 5 years, $15 \%$ at 10 years, and $0 \%$ at 15 years, which points to the poor outcomes of the tumor [3]. Due to the poor understanding of the mechanism of SACC progression, no effective therapy has been provided [5]. Therefore, it is impending to define the underlying mechanism of SACC and to explore the methods for SACC treatment. 
$\mathrm{NOTCH}$ signaling critically influences cellular differentiation and proliferation, embryonic development and blood vessel formation [6]. Disordered NOTCH signaling affects the occurrence, development, invasion and metastasis of many different types of tumors, as well as inducing tumors directly or indirectly via interactions with other signaling pathways [7-9]. Multiple factors of $\mathrm{NOTCH}$ signaling, including ligands, receptors, signal transducers and effectors execute pleiotropic effects [10]. In our previous study, we explored the role of NOTCH1 [11] and its primary effector HES1 in SACC. HEY is normally considered another primary target of $\mathrm{NOTCH}$ signaling as well as a related but distinct member of the bHLH family compared with HES, which can also play a role as a transcriptional repressor in cell fate decisions $[9,10,12,13]$. The HEY family consists of HEY1, HEY2 and HEYL, which are encoded by three distinct genes with similar structures [14] and encode a nuclear protein belonging to the hairy and enhancer of split-related (HESR) family of basic helix-loop-helix (bHLH)-type transcriptional repressors.

Although increasing advances toward the function of HEY1 have been reported in electronic public literature searches, the mechanism of HEY1 in SACC remains uncovered. Our group used a siRNA technique to inhibit the expression of HEY1 in SACC cells on account of the findings of RNA-Seq. Through a series of experiments in vitro and in vivo, we aimed to illuminate the functional role of the NOTCH1HEY1 pathway in salivary adenoid cystic carcinoma, which will open a new avenue to our understanding of the NOTCH signaling pathway in SACC.

Table 1. The sequences of the siRNAs used in the current study

\begin{tabular}{|c|c|c|}
\hline Name & Sequence & \\
\hline siRNA-HEY1-602 & $\begin{array}{l}\text { 5'-CGCGUUAUCUGAGC } \\
\text { AUCAUTT-3' }\end{array}$ & $\begin{array}{l}\text { 5'-AUGAUGCUCAGAUA } \\
\text { ACGCGTT-3' }\end{array}$ \\
\hline siRNA-HEY1-1071 & $\begin{array}{l}\text { 5'-GGCAAGCCCUAUAG } \\
\text { ACCUUTT-3' }\end{array}$ & $\begin{array}{l}\text { 5'-AAGGUCUAUAGGGC } \\
\text { UUGCCTT-3' }\end{array}$ \\
\hline siRNA-HES1-670 & $\begin{array}{l}\text { 5'-CCAACUGCAUGACC }^{\text {CAGAUTT-3' }}\end{array}$ & $\begin{array}{l}\text { 5'-AUCUGGGUCAUGCA } \\
\text { GUUGGTT-3' }\end{array}$ \\
\hline NC & $\begin{array}{l}\text { 5'-UUCUCCGAACGUGU } \\
\text { CACGUTT-3' }\end{array}$ & $\begin{array}{l}\text { 5'-ACGUGACACGUUCG } \\
\text { GAGAATT-3' }\end{array}$ \\
\hline
\end{tabular}

\section{Materials and Methods}

\section{Cell culture and clinical samples}

The SACC-LM cell line was obtained from the Peking University Health Science Center. The cells were maintained in RPMI-1640 medium (Gibco BRL, Grand Island, NY) supplemented with 10\% fetal bovine serum (Gibco) and incubated in a humidified atmosphere of $95 \%$ air and $5 \% \quad \mathrm{CO}_{2}$ at $37^{\circ} \mathrm{C}$. Experiments were performed using cells in the exponential phase of growth. Tissue samples were obtained from Fuzhou PLA General Hospital and Fujian Medical University Union Hospital. Seventy-seven normal salivary tissues and eithty-seven SACC samples were included. This study was approved by the Institutional Review Board of Fujian Medical University, and written informed consent was obtained from each participant.

\section{RNAi transfection}

The negative control (NC) siRNA and two siRNAs against HEY1 were synthesized (GenePharma, Shanghai, China). The siRNA sequences are listed in Table 1. Cells were transfected with siRNAs using Lipofectamine RNAiMAX (Invitrogen, USA) according to the manufacturer's instructions.

\section{Co-transfection of plasmid DNA and siRNA}

The pcDNA3.1-NICD1 plasmid was a kind gift from Dr. Glenn Doughty, Harvard Medical School [15]. The co-transfection combinations are the vector plasmid and siRNA NC (named: VE NC), the vector plasmid and siRNA targeted HEY1 (named: VE siRNA-HEY1), the vector plasmid and siRNA targeted HES1 (named: VE siRNA-HES1), the pcDNA3.1-NICD1 plasmid and siRNA NC (named: N1 NC), the pcDNA3.1-NICD1 plasmid and siRNA targeted HEY1 (named: N1 siRNA-HEY1) and the pcDNA3.1-NICD1 plasmid and siRNA targeted HES1 (named: N1 siRNA-HES1). Cells were transfected with plasmid DNA and siRNAs at the same time using Lipofectamine 2000 (Invitrogen, USA) according to the manufacturer's instructions.

\section{Inhibitor of Mastermind Recruitment-1 (IMR-1) treatment}

The inhibitor of Mastermind Recruitment-1 (IMR-1) purchased from MedChemExpress (New Jersey, USA) was dissolved in DMSO (dimethyl sulfoxide) at a concentration of $10 \mathrm{mM}$, and stored at $-20^{\circ} \mathrm{C}$. For in vitro experiment, seeded cells to be $30-$ $40 \%$ confluent, the cells were treated with $10 \mu \mathrm{M}$ and $20 \mu \mathrm{M}$ IMR-1 at indicated time. The DMSO as a vehicle was used as a negative control.

\section{Immunohistochemistry}

For the immunohistochemical assays, 5 - $\mu$ m-thick tissue sections were mounted onto slides coated with poly-L-lysine. After deparaffinization in xylene, the sections were rehydrated in a decreasing gradient of ethanol and washed for $10 \mathrm{~min}$ in phosphate-buffered saline (PBS) ( $\mathrm{pH}$ 7.2). Endogenous peroxidase activity was inhibited by incubation in methanol containing $3 \% \mathrm{H}_{2} \mathrm{O}_{2}$ for $10 \mathrm{~min}$. After several washes in PBS, the sections were blocked with a universal blocking reagent (Maxin, USA) for $10 \mathrm{~min}$ at room temperature 
and then incubated with primary antibodies against Caspase-3 (1:300, Cell Signaling, USA), Caspase-9 (1:500 dilution, Abcam, UK), Ki-67 (1:500 dilution, Abcam, UK), NOTCH1 (1:400 dilution, Sigma, USA) and HEY1 (1:30 dilution, Abcam, UK) for $1 \mathrm{~h}$ at room temperature. After several washes in PBS, the sections were incubated with a biotin-conjugated secondary antibody (Maxin) for $10 \mathrm{~min}$ at room temperature. After several washes in PBS, the sections were incubated with streptavidin-peroxidase (Maxin) for 10 $\mathrm{min}$ at room temperature. The sections were rinsed with PBS, and the antibody complexes were visualized by incubation with diaminobenzidine tetrahydrochloride (DAB) chromogen (Maxin). The sections were then counterstained with hematoxylin (Dako, Denmark), dehydrated, and examined by light microscopy. All slides were reviewed independently by two pathologists who were blinded to each other's readings. The staining results were assessed on a three-tier scale: negative indicated no staining, 1+ indicated weak staining and $2+$ indicated strong staining. Immunohistochemical results were graded with 3 different scores (negative, positive and strong positive) as follows: negative indicated no staining or $1+$ staining in $\leq 30 \%$ of cells, positive indicated $1+$ staining in $>30 \%$ of cells or $2+$ staining in $<50 \%$ of cells and strong positive indicated $2+$ staining in $>50 \%$ of cells.

\section{Quantitative real-time PCR analysis}

Total RNA was extracted from cells with Trizol reagent (Invitrogen, USA) and reverse transcribed into cDNA with the PrimeScript RT reagent kit (TaKaRa, Japan). The cDNA was used as the template to detect the expression of the genes of interest by qRT-PCR with SYBR Premix Ex Taq ${ }^{\mathrm{TM}}$ (TaKaRa, Japan). The primers used in this study are listed in Table 2. Data were analyzed according to the $2^{-\Delta \Delta} \mathrm{Ct}$ method.

\section{Western blot assay}

Total protein was separated by $8 \%$ SDS-PAGE and transferred onto PVDF membranes (Amersham, USA). Subsequently, the membranes were immunoblotted with primary antibodies against E-Cadherin (1:1000 dilution, Cell Signaling, USA), N-Cadherin (1:1000 dilution, Cell Signaling, USA), Twist1 (1:1000 dilution, Cell Signaling, USA), NOTCH1 (1:1000 dilution, Sigma, USA), HES1(1:1000 dilution, Abcam, UK) and HEY1 (1:250 dilution, Abcam, UK) and $\beta$-actin (1:2000 dilution, Sigma, USA) in $5 \%$ bovine serum albumin overnight, washed three times with Tris-buffered saline containing $0.1 \%$ Tween 20, and incubated with the secondary antibody (1:2000 dilution, Boster, China). The immunoreactive protein bands were visualized using CDP STAR reagent (Roche, IN, USA), and signals were scanned with a densitometer for the semiquantification of signal intensity.

\section{Cell viability assay}

Cell proliferation was measured by counting viable cells with a Cell Counting Kit-8 (Dojindo, Kumamoto, Japan). Cells were first transfected with the indicated siRNA for $24 \mathrm{~h}$ and then planted into a 96-well plate. In the experiment of small molecule inhibitors for the treatment of SACC cell, the cells were seeded into a 96-well plate for $16 \mathrm{~h}$, then treated with different doses of inhibitor IMR-1. At the same time each day for 5 consecutive days, the original culture medium was removed, and $10 \mu \mathrm{l}$ of CCK8 and $90 \mu \mathrm{l}$ of fresh RPMI-1640 medium were added to each well. The cells were incubated at $37^{\circ} \mathrm{C}$ for $1 \mathrm{~h}$. The absorbance of each well was measured with a microplate reader (Pharmacia Biotech, USA) at $450 \mathrm{~nm}$.

\section{Colony formation assay}

Twenty-four hours after siRNA transfection, the cells were plated into $6-\mathrm{cm}$ plates (600 cells per plate) and cultured for 2 weeks. In the experiment of small molecule inhibitors for the treatment of SACC cell, the cells were seeded into a 6-well plate for $16 \mathrm{~h}$, then treated with different doses of inhibitor IMR-1 and cultured for 2 weeks. Colonies were fixed with cold methanol for $10 \mathrm{~min}$ and stained with $1 \%$ crystal violet for $30 \mathrm{~min}$.

\section{In vitro cell invasion assay}

Cell invasion was determined using 24-well Matrigel-coated transwell chambers $(8-\mu \mathrm{m}$ pore size, BD Science, USA). Twenty-four hours after siRNA transfection, cells were serum starved for $24 \mathrm{~h}$ and then collected in RPMI-1640 medium containing 1\% FBS. Cells were plated in the upper chamber at a density of $1.0 \times 10^{5}$ cells per well, and $800 \mu \mathrm{l}$ of RPMI-1640 medium containing 10\% FBS was added to the lower chamber. After incubation at $37^{\circ} \mathrm{C}$ for $48 \mathrm{~h}$, the Matrigel and cells in the upper chamber were removed using a cotton swab and stained with $1 \%$ crystal violet for $10 \mathrm{~min}$. Cells were counted and photographed by microscopy in at least five random fields $(\times 200)$.

\section{In vitro cell migration assay}

Cell migration assays were performed using 24-well transwell chambers $(8-\mu \mathrm{m}$ pore size, BD Science, USA). The procedure used for this assay was similar to that of the cell invasion assay, except the transwell was not coated with Matrigel. 
Table 2. The primers for real-time PCR and semiquantitative RT-PCR used in the current study

\begin{tabular}{|c|c|c|}
\hline Gene & Forward & Reverse \\
\hline ACTB & 5'-CCTGGCACCCAGCACAAT-3' & 5'-GGGCCGGACTCGTCATACT-3' \\
\hline HEY1 & 5'-CGAGGTGGAGAAGGAGAGTG-3' & 5'-CTGGGTACCAGCCTTCTCAG-3' \\
\hline NOTCH1 & 5'-GGAAGTTGAACGAGCATAGTCC-3' & 5'-GCATGATGCCTACATTTCAAGA-3' \\
\hline TNNT3 & 5'-ATTCGTGCAGAGAAGGAGAGGG-3' & 5'-TCTTGCCTCTCTTCTGGTCAGC-3' \\
\hline IFI6 & 5'-CTACCTGCTGCTCTTCACTTGC-3' & 5'-TCCTCCGACGGCCATGAAG-3' \\
\hline RELB & 5'-CGTGCATGCTTCGGTCTGG-3' & 5'-GCCGTTCTCCTTGATGTACTCG-3' \\
\hline KRT-17 & 5'-AGGACAATTGAGGAGCTGCAGA-3' & 5'-ATTGATGTCGGCCTCCACACT-3' \\
\hline MMP7 & 5'-CATGAGTGAGCTACAGTGGGA-3' & 5'-CTATGACGCGGGAGTTTAACAT-3' \\
\hline TPD52 & 5'-CTGTTGGCTCAGTCATCACCAA-3' & 5'-CTTTTCTGGAAGAGGCTCCGTG-3' \\
\hline ABHD3 & 5'-TACCCTTCTGCTCCTTTCCTGG-3' & 5'-TCCAATGACTCTGAGCAAGCGA-3' \\
\hline Snail1 & 5'-GCCTTCAACTGCAAATACTGC-3' & 5'-CTTCTTGACATCTGAGTGGGTC-3' \\
\hline Twist1 & 5'-CCATGTCCGCGTCCCACTA-3' & 5'-CCCACGCCСТGTTTCTTTGAAT-3' \\
\hline Fibronectin 1 & 5'-GCCGAGGTTTTAACTGCGAGAG-3' & 5'-CGATGCAGGTACAGTCCCAGAT-3' \\
\hline MMP1 & 5'-GGGGAGATCATCGGGACAACTC-3' & 5'-AGAATGGCCGAGTTCATGAGCT-3' \\
\hline MMP2 & 5'-CAAGGACCGGTTCATTTGGC-3' & 5'-GGCCTCGTATACCGCATCAA-3' \\
\hline MMP3 & 5'-TCAGAACCTTTCCTGGCATCCC- $3^{\prime}$ & 5'-CAGCCTGGAGAATGTGAGTGGA-3' \\
\hline MMP9 & 5'-GCCCGACCCGAGCTGACTC-3' & 5'-TTCAGGGCGAGGACCATAGAGG-3' \\
\hline MMP11 & 5'-CCGTGCTGACATCATGATCGAC-3' & 5'-CAAATTCATGGGCTGCCACCTG-3' \\
\hline MMP13 & 5'-TTTCAACGGACCCATACAGTTTG-3' & 5'-CATGACGCGAACAATACGGTTA-3' \\
\hline
\end{tabular}

\section{Cell apoptosis assay}

Cellular apoptosis was analyzed using a FITC Annexin V Apoptosis Detection Kit (BD Pharmingen ${ }^{\mathrm{TM}}$, USA). At $48 \mathrm{~h}$ posttransfection, the cells were collected and washed in PBS and then stained with annexin $\mathrm{V}$ and propidium iodide for 15 min. The percentage of apoptotic cells was quantified using a BD FACS Verse flow cytometer.

\section{D Spheroid formation assay}

A tumor spheroid formation assay was performed using ultra-low attachment (ULA) 6-well round-bottomed plates (Corning, USA). Twenty-four hours after siRNA transfection, the cells were seeded at a density of 40000 cells per well in $4 \mathrm{ml}$ of DMEM/F12 without FBS in a ULA plate. The 3D tumor spheroids were incubated for 14 days before analysis.

\section{Establishment of a xenograft tumor model}

Female BALB/C nude mice 6-8 weeks of age were purchased from the Center for Animal Experiments of Fujian Medical University. Cells (2× $10^{6}$ ) were suspended in $0.2 \mathrm{ml}$ of serum-free DMEM and injected into the right axillary fossa of each mouse. Tumor size was calculated using the formula $\mathrm{V}=$ width $^{2} \times$ length $/ 2$. At the end of the experiment, the tumors were harvested and weighed. The experimental animal protocols were approved by the Animal Care and Use Committee of Fujian Medical University.

\section{Statistical analysis}

The statistical analysis of HEY1 immunoreactivity was performed using the rank-sum test. The statistical analysis of PCR results and the in vitro cell migration/invasion assays was determined by One-Way ANOVA followed by Tukey's multiple Comparison test. $p<0.05$ was considered statistically significant and is indicated in the figures as ns when $\mathrm{P}>0.05$, * when $\mathrm{P}<0.05$, ** when $\mathrm{P}<0.01$ and ${ }^{* * *}$ when $\mathrm{P}<0.001$.

\section{Results}

\section{The expression of HEYI is regulated by NOTCHI in SACC cells}

We previously reported that NOTCH1 and its downstream gene HES1 both act as an oncogene in SACC and accelerate the proliferation and migration of SACC cells [16]. We performed and analyzed the RNA-Seq results pertaining to NOTCH1 upregulation in SACC cells, and another NOTCH1 downstream gene, HEY1, drew our attention. We validated satisfactory RNA-Seq results and deposited the data in the NCBI Sequence Read Archive (SRA, https://www.ncbi.nlm.nih.gov/sra) under accession no. SRR5572289, which revealed a total of 1323 coding genes that displayed differential expression in NOTCH1-overexpressing cells. In addition, Gene Ontology (GO) analysis provided a description of cell function: the highly expressed genes are involved in cell mobility, cell differentiation, proliferation and signal transduction, while the weakly expressed genes are involved in metabolism, transport and differentiation.

On account of the RNA-Seq results, another 8 genes were randomly selected along with NOTCH1, and qRT-PCR was performed to verify their expression. The expression levels of HEY1, TNNT3, IFI6, RELB, KRT-17, MMP7, TPD52 and ABHD3 


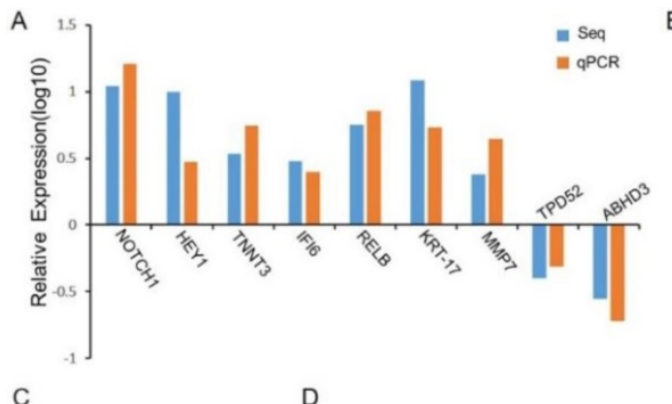

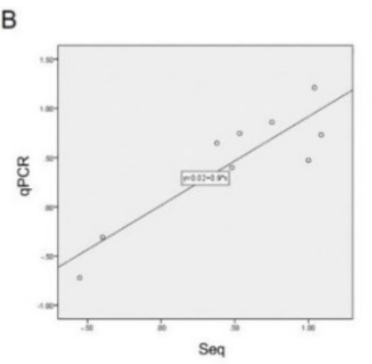

$\mathrm{F}$

E

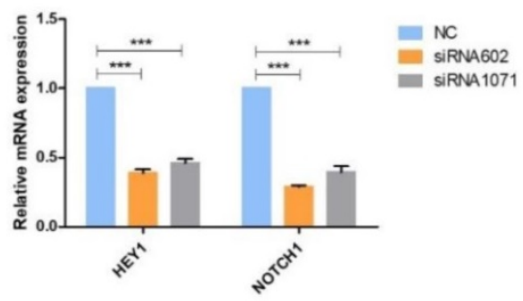

G
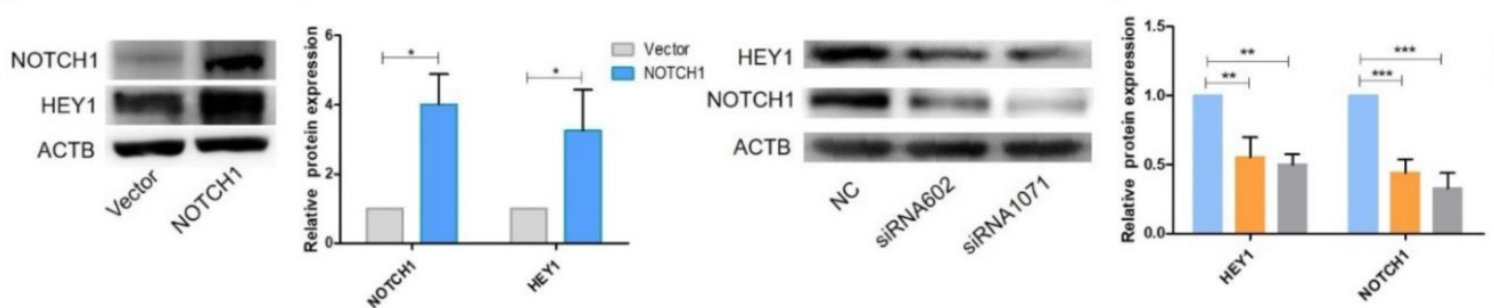

Figure 1. The NOTCH1-HEY1 pathway is a positive feedback loop in SACC cells. (A) The expression of NOTCH1, HEY1, TNNT3, IFI6, RELB, KRT-17, MMP7, TPD52 and ABHD3 genes, which were selected from the RNA-Seq results, was measured by qRT-PCR. (B) Linear regression analysis was used to compare the expression levels of the abovementioned genes between the RNA-Seq and qRT-PCR results. (C, D) Western blot analysis and the quantification of NOTCHI and HEY 1 expression in SACC cells after NOTCH1 upregulation by PCDNA3.1-NICDI plasmid transfection. (E) The mRNA expression of NOTCH1 and HEY1 after HEY1 downregulation by siRNAs was detected by RT-PCR. (F, G) The protein expression of NOTCH1 and HEY1 when HEY1 inhibition was measured by western blot analysis. *P $<0.05$, ** $\mathrm{P}<0.01$, and $* * * \mathrm{P}<0.005$.

obtained by qPCR were consistent with the RNA-Seq results (Fig. $1 \mathrm{~A}, \mathrm{P}<0.001$ ), which suggested that HEY1 is a specific downstream gene of the NOTCH1 signaling pathway. Linear regression analysis of the relationship between RNA-Seq and qPCR revealed a reliable RNA-Seq result (Fig. $1 B, R^{2}=0.805$ ). Next, we employed western blot analysis to examine the expression of HEY1 in SACC cells (Fig. 1C) and found that the expression of HEY1 was significantly enhanced in the group in which NOTCH1 was overexpressed compared with the control group. In addition, as described in our previous qPCR results, HEY1 expression is downregulated when NOTCH1 is restrained in SACC cells [11]. Taken together, these data suggest that NOTCH1 is significantly associated with the expression of HEY1.

\section{HEY 1 inhibition decreases NOTCHI expression in SACC cells}

It has been reported that all NOTCH receptors were significantly associated with HEY1, and HEY1 could reciprocally regulate the expression of NOTCH4 in HNSCC [17]. Therefore, we wondered whether HEY1 also has this molecular mechanism that reversely regulated NOTCH1 in SACC. We applied siRNA-mediated knockdown of HEY1 in SACC-LM cells in the following experiments. As shown by real-time RT-PCR (Fig. 1E) and western blot (Fig. 1F-G) analyses, siRNAs targeting HEY1 (siRNA-602 and siRNA-1071) efficiently reduced the expression of HEY1 in SACC-LM cells compared with the negative control (NC). In previous studies, it was reported that the expression of HEY1 was regulated by NOTCH1 receptors [18-19]. However, we respectively determined the mRNA and protein levels of NOTCH1 in SACC-LM cells after HEY1 was suppressed by siRNAs through real-time PCR (Fig. 1E) and western blotting (Fig. 1F-G). The results showed that the expression of NOTCH1 was obviously reduced. Thus, these results indicate that HEY1 may reverse regulate NOTCH1 in SACC cells, unlikely previous reports. Therefore, we focused on the effector of NOTCH signaling, HEY1, for a series of further studies.

\section{NOTCH1-HEY 1 is upregulated in human adenoid cystic carcinoma tissues}

The immunohistochemistry results of NOTCH1 and HES1 in our previous study demonstrated that higher expression levels were related to metastatic and recurrent SACC. As another pivotal member of the NOTCH1 signaling path, we investigated the expression of HEY1 in salivary adenoid cystic carcinoma, including 77 samples of normal tissue and 87 adenoid cystic carcinoma cases, via immunohistochemistry. According to the staining results, we classified the expression of HEY1 into a four-tier scale. As shown in Fig. 2A and 2B, HEY1 expression was absent or low in normal salivary gland tissues, while higher expression levels were observed in the adenoid cystic carcinoma cases $(\mathrm{P}<0.001)$. Then, we would like to further confirm whether there was a positive correlation between NOTCH1 and HEY1 in salivary adenoid cystic carcinoma tissues. Immunohistochemistry was used to detect the expression of NOTCH1 and HEY1 in 27 cases of the adenoid cystic 
carcinoma tissues. The results showed that the expression of NOTCH1 and HEY1 were positively correlated in the salivary adenoid cystic carcinoma tissues (Fig. 2C, $\mathrm{P}<0.01, \mathrm{n}=27$ ). In conclusion, these results indicated that the NOTCH1-HEY1 signaling pathway was specifically up-regulated in SACC.

\section{HEYI regulates cellular apoptosis}

To explore the functional effects of HEY1 on the proliferation of cancer cells, the cells were transfected with siRNAs, and the growth of SACC-LM cells was significantly inhibited in the siRNA602 and siRNA1071 groups according to the CCK8 assay (Fig. $3 \mathrm{~A}, \mathrm{P}<0.001$ at days 3, 4 and 5). Additionally, the same results were detected in colony formation assays (Fig. $3 \mathrm{~B}, \mathrm{P}<0.01, \mathrm{n}=3$ ). Furthermore, we knocked down HEY1 by siRNA transfection for $48 \mathrm{~h}$ and then quantified apoptotic cells using annexin $\mathrm{V}$ and PI staining and flow cytometric analysis. Compared with the negative control cells (Fig. 3C), after $48 \mathrm{~h}$ of transfection, the percentage of both early apoptosis (annexin V-positive and PI-negative) and late apoptosis (annexin V-positive and PI-positive) cells was increased in HEY1-silenced cells. These assays collectively support that HEY1 plays an oncogenic role in SACC.
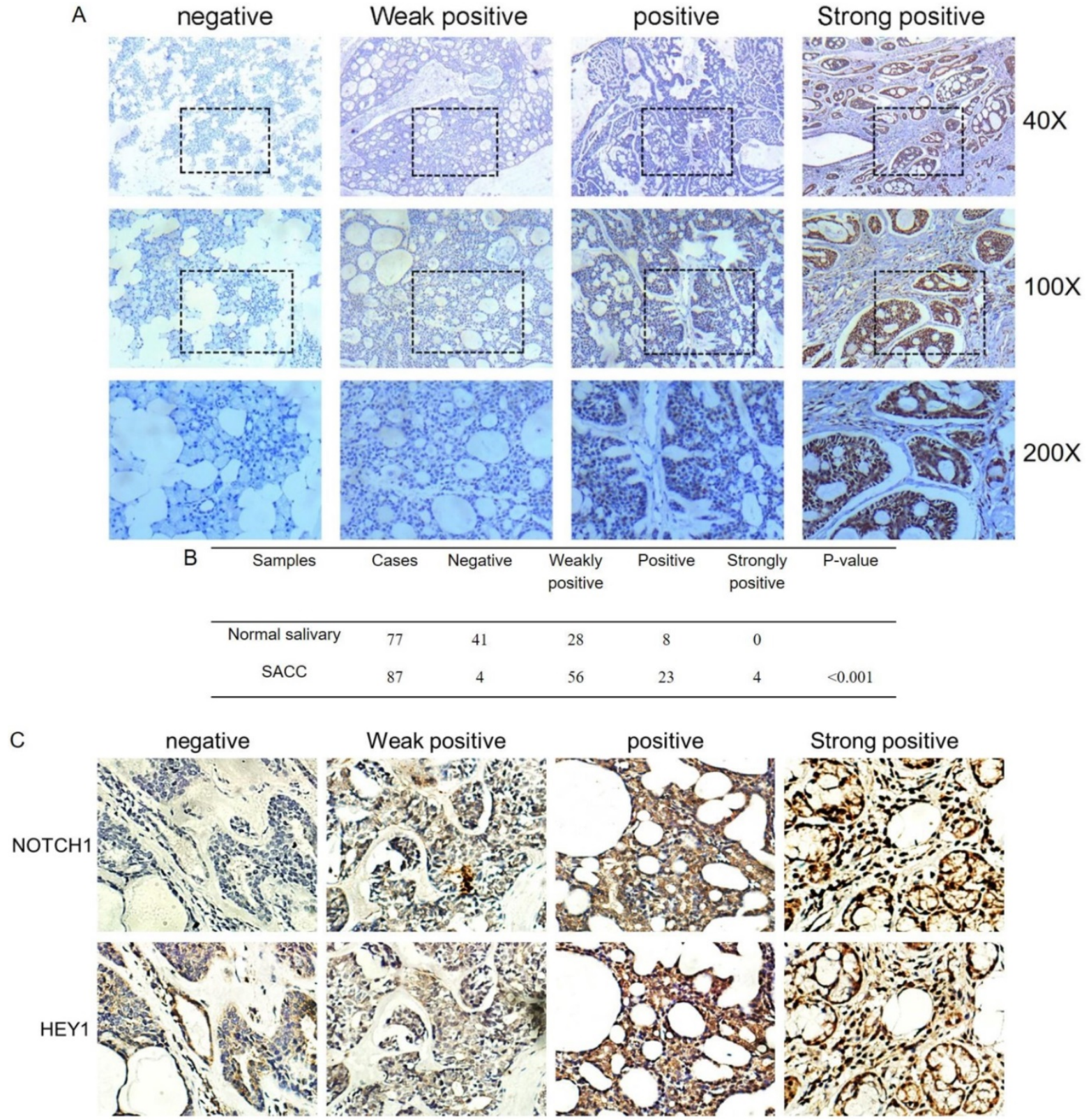

Figure 2. HEY1 is upregulated in adenoid cystic carcinoma tissues and is positively correlated with NOTCH1. (A) Representative images of HEY1 expression, as determined by immunohistochemistry and the following four-tier scale: negative, weakly positive, positive and strongly positive. (B) The statistics of the expression of HEYl in adenoid cystic carcinoma tissues and normal tissues. (C) Representative images of NOTCHI and HEYl expression, as determined by immunohistochemistry and the following four-tier scale: negative, weakly positive, positive and strongly positive. 


\section{IMR-I inhibits the proliferation of SACC cell in vitro}

In the above experiments, we found that knockdown of HEY1 was effectively inhibited the proliferation of SACC cells. So, we wondered whether the inhibitory effect of knockdown of HEY1 on SACC cells was consistent with the NOTCH signaling inhibitor. Inhibitor of Mastermind Recruitment-1 (IMR-1) is a new class of NOTCH pathway inhibitor that target transcriptional activation [20]. The results of CCK8 assay (Fig. 3D, P<0.001 at days 2, 3, 4, 5 and 6) and colony formation assay (Fig. $3 E, P<0.01, n=3$ ) showed that treatment of SACC cells with IMR-1 displayed a dose-dependent reduction. The NOTCH signaling pathway inhibitor IMR-1 can effectively inhibit the growth of SACC cells, which is consistent with the effect of HEY1-specific siRNAs on cell proliferation. Therefore, it is demonstrated from another perspective that HEY1 regulates the proliferation of SACC cells through the $\mathrm{NOTCH}$ signaling pathway.

\section{HEYI knockdown inhibits SACC cell spheroid formation and growth in vitro}

Some studies have shown that spheroids of cancer cells, because of their morphological and biological characteristics that are similar with those of solid tumors, are a promising model for the simulation of tumor changes in vivo [21]. The results of the tumor cell spheroid formation assay showed that the volume (Fig. 3F) and number (Fig. 3G) of tumor spheroids were decreased in the inhibition group compared to the control group. Furthermore, we also detected the mRNA expression of several stemness-related genes, and the results showed that the knockdown of HEY1 in SACC-LM cells inhibited the expression of OCT4, ALDH1, Snail1, Twist1 and Fibronectin 1 (Fig. 3H). Taken together, these results suggest that the knockdown of HEY1 can effectively inhibit 3D tumor cell spheroid formation and stemness in SACC stem cells.
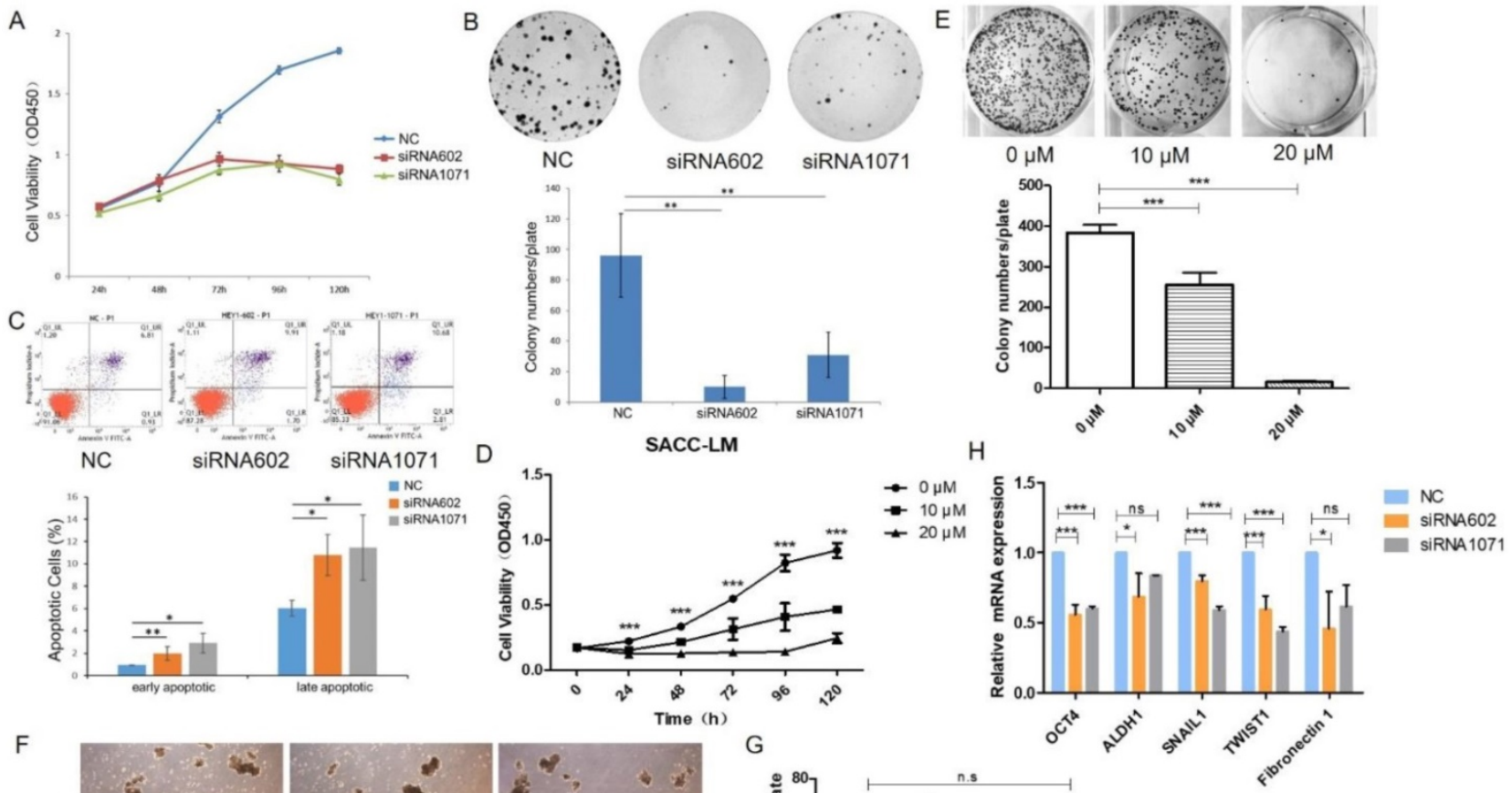

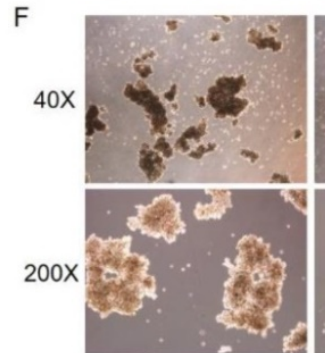

NC

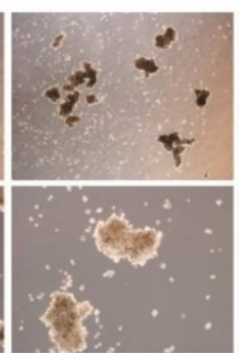

SiRNA602

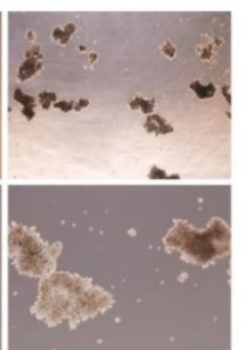

siRNA1071

G

Figure 3. HEYI promotes cell proliferation, self-renewal and regulates cellular apoptosis, and IMR-1 inhibits cell growth. After siRNA transfection, the cell proliferation was measured by CCK8 (A, P<0.001 by One-Way ANOVA followed by Tukey's multiple Comparison test from days 3,4 and 5 ) and colony formation assays (B, $\mathrm{P}<0.01$ by One-Way ANOVA followed by Tukey's multiple Comparison test, $n=3)$. The percentages of early and late apoptosis cells were detected by flow cytometry $(C, P<0.05$ by One-Way ANOVA followed by Tukey's multiple Comparison test). After treated with IMR-1, the cell proliferation was measured by CCK8 (D, P<0.001 by One-Way ANOVA followed by Tukey's multiple Comparison test from days 2, 3, 4 and 5) and colony formation assays (E, P<0.01 by One-Way ANOVA followed by Tukey's multiple Comparison test, $n=3$ ). (F) Representative images of tumor spheroids of the NC, siRNA602 and siRNA1071 groups. The numbers of cell spheroids in the NC, siRNA602 and siRNA1071 groups (G, P<0.05 by One-Way ANOVA followed by Tukey's multiple Comparison test). $(H)$ The mRNA expression of OCT4, ALDH1, Snail1, Twist 1 and Fibronectin 1 when HEY1 was downregulated in SACC cells. $* \mathrm{P}<0.05$, $* * \mathrm{P}<0.01$, and $* * * \mathrm{P}<0.005$. 

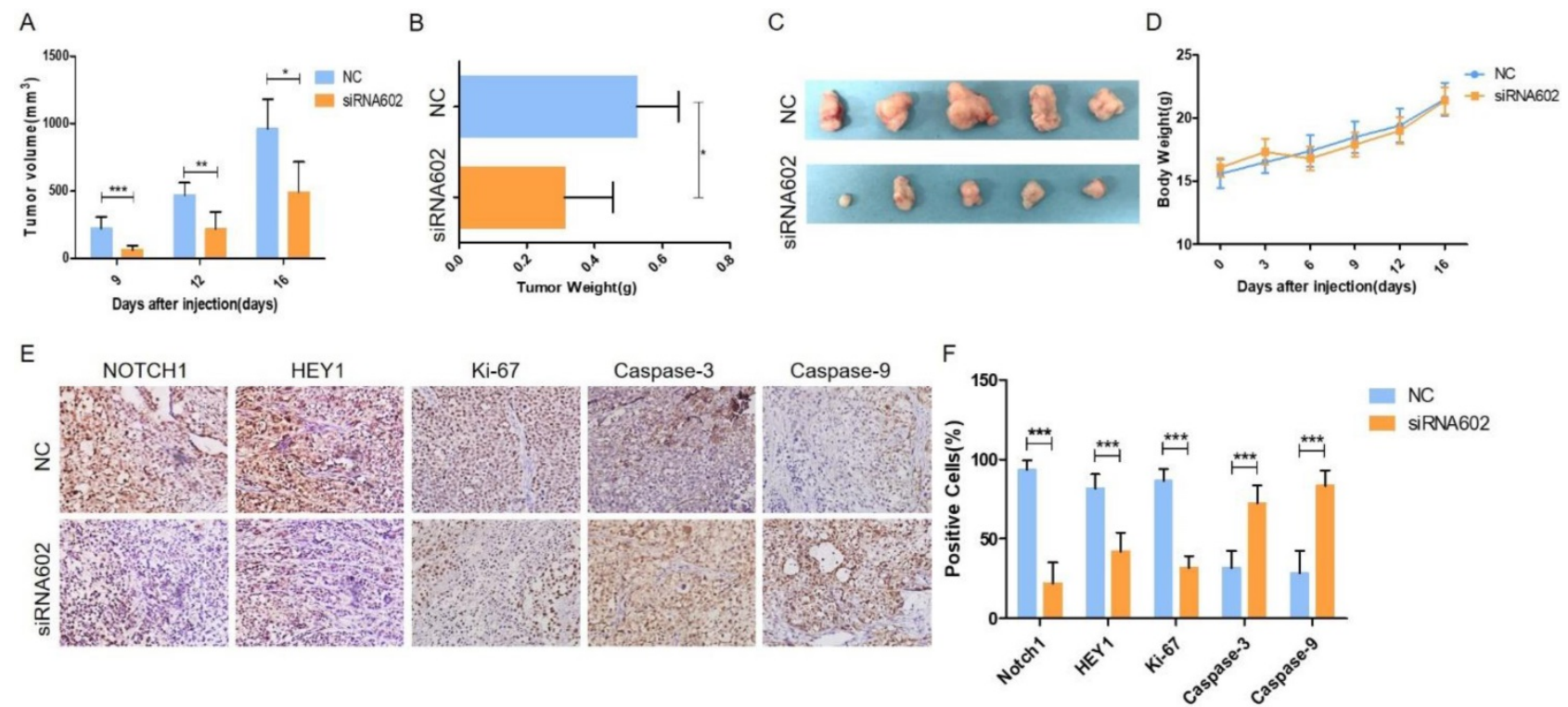

Figure 4. Knockdown of HEYI inhibits the growth of tumors in vivo. (A) Changes in the tumor volume of nude mice were measured during the experiment. The nude mice were sacrificed, and the tumors were removed, weighed (B, P $<0.05$ by t-test) and photographed (C). Changes in the body weights of nude mice in the NC and siRNA602 groups (D, P $>0.05$ at days $0,3,6,9,12$ and 16 by t-test). (E) The expression of NOTCH1, HEY1, Ki-67, Caspase- 3 and Caspase- 9 was detected in the xenograft tumors by IHC (DAB, 200x), and the positive cells were counted and compared among the groups $(\mathrm{F}, \mathrm{P}<0.001$ by t-test). $* \mathrm{P}<0.05$, $* * \mathrm{P}<0.01$, and $* * * \mathrm{P}<0.005$.

\section{Knockdown of HEY 1 inhibits tumorigenicity in vivo}

In vitro cell studies demonstrated that downregulating the expression of HEY1 in SACC-LM cells can inhibit cell proliferation, promote cell apoptosis, block the cell cycle, and inhibit spheroid formation. To further study the oncogenic effect of HEY1 on tumorigenicity in vivo, SACC-LM cells were separately transfected with different siRNAs (NC and siRNA-602) and then subcutaneously injected into the flanks of nude mice. Throughout the experimental observation period, we weighed the nude mice every 3 days and measured the length and width of the subcutaneous tumor. As seen from the trend of tumor growth (Fig. 4A), the tumor growth rate of the siRNA602 group was significantly lower than that of the NC group, and the weight of nude mice was not obviously changed (Fig. 4D). Moreover, the wet weight (Fig. 4B) and size (Fig. 4C) of the xenograft tumors of the siRNA602 group were significantly decreased compared to those of the NC group. Additionally, the expression of NOTCH1, HEY1, Ki-67, Caspase-3 and Caspase-9 in the xenograft tumors was detected by immunohistochemistry. The expression of NOTCH1 and HEY1 were significantly decreased in the siRNA602 group. The proliferation index (Ki-67) of xenograft tumors of the siRNA-602 group was decreased, while the expression of apoptosis-related proteins (Caspase-3 and Caspase-9) was increased compared with that in the NC group (Fig. 4E, 4F). Collectively, the above results indicate that the inhibition of HEY1 expression in salivary adenoid cystic carcinoma cells can obviously repress tumor formation and growth and induce cell apoptosis in vivo.

\section{HEY 1 increases cell migration and invasion in vitro}

Next, we examined the migration and invasion of SACC cells with reduced expression of HEY1. As shown in Fig. 5, transfection of the HEY1-specific siRNAs into SACC-LM cells significantly inhibited cell motility and invasion, as indicated by the wound-healing (Fig. 5A) and transwell (Fig. 5B) assays. These findings are concordant with the idea that HEY1 might act as an oncogene in SACC by contributing to the migration and invasion of SACC cells.

\section{HEY1 regulates the Epithelial-Mesenchymal Transition and MMPs expression in SACC cells}

We further explored the underlying molecular mechanisms related to HEY1 and SACC biological properties. It has been reported that HEY1 expression is associated with the epithelial-mesenchymal transition (EMT) [22]. Tumor cells need to undergo partial or full epithelial-mesenchymal transition and are transformed into migrating and invasive cells [23]. In cancer, the EMT is associated with tumorigenesis, invasion, metastasis, tumor stemness and resistance to therapy [24]. Therefore, we measured the expression of EMT-related genes when HEY1 was inhibited in SACC cells (Fig. 5C, 5D). The expression of a mesenchymal marker (N-cadherin) was decreased in the siRNA602 and siRNA1071 groups, while the 
epithelial marker E-cadherin was significantly increased compared to the NC group. The transcription factor Twist1, which is known to promote tumor metastasis and induce the EMT, was obviously reduced when HEY1 was knocked down. In addition, we also detected the expression of matrix metalloproteinases (MMPs) in SACC cells transfected with different siRNAs (NC, siRNA602 and siRNA1071). The results showed that the knockdown of HEY1 in SACC-LM cells suppressed the expression of MMP1, MMP2, MMP3, MMP9, MMP11 and MMP13 (Fig. 5E). Thus, these results reveal that HEY1 activation drives the EMT and promotes the expression of MMPs in SACC.

\section{HEY 1 and HESI contribute equally in mediating Notch 1 signaling pathway in SACC cells}

We have demonstrated that HEY1 and HES1 [16] could promote proliferation, invasion and migration in SACC. They both played an important role in the NOTCH signaling pathway. Therefore, we wondered which would play more important role of NOTCH1 oncogenic function in SACC? As shown in Fig. 6A, after co-transfection of the plasmid DNAs and siRNAs into SACC-LM cells, the expression of NOTCH1, HEY1 and HES1 were significantly elevated in the N1 NC group while compared with the VE NC group. NOTCH1 was obviously inhibited in the condition of HEY1 or HES1 being knockdown in the N1 siRNA-HEY1 group or in the N1 siRNA-HES1 group compared with N1 NC group. Interestingly, the expression of HEY1 was also visibly reduced in the HES1 knockdown group. However, in the HEY1 knockdown group, the same phenomenon was not observed. We speculated that HES1 might regulate the biological behavior of SACC cells by affecting the expression of HEY1 in the NOTCH signaling pathway. The CCK8 (Fig. 6B) showed that the cell proliferation in the N1 NC group was enhanced compared with other groups $(\mathrm{P}<0.05$ at days 2, 3, 4, 5, 6). Compared with the N1 NC group, the cell growth of the N1 siRNA-HEY1 group and the N1 siRNA-HES1 group decreased, but there was no significant difference in the growth inhibition of these two groups (N1 siRNA-HEY1 group vs N1 siRNA-HES1 group, $\mathrm{P}>0.05$ at days $2,3,5$ and $6, \mathrm{P}<$ 0.05 at day 4). As expected, the colony formation assays (Fig. 6C) also had the same performance. Moreover, the invasion and migration of SACC cells were boosted in the N1 NC group, and the cell motility and invasion were inhibited in the N1 siRNA-HEY1 group and the N1 siRNA-HES1 group. However, there was no obviously difference in these two groups (Fig. 6D). Taken together, HEY1 and HES1 showed equal importance as a carcinogen in the NOTCH signaling pathway.
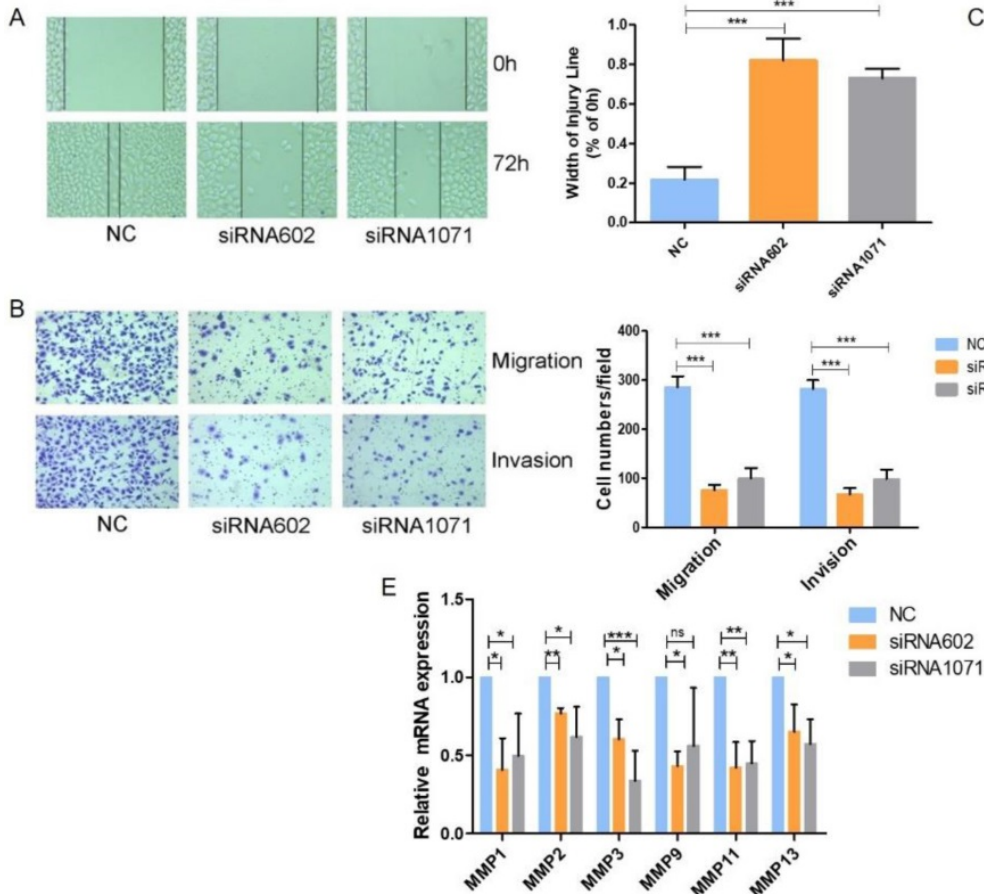
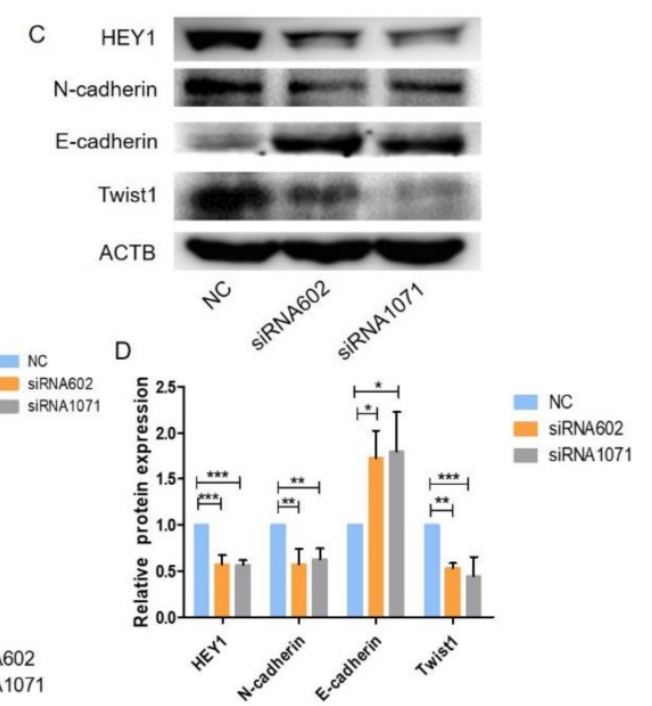

Figure 5. HEY1 knockdown inhibits SACC cell migration and invasion, and regulates the epithelial-mesenchymal transition and MMP expression in SACC cells. After inhibited the exression of HEY1, the cell migration and invasion were measured by, wound healing assay (A) and transwell assay coated with or without matrigel(B, $\mathrm{P}<0.001$ by One-Way ANOVA followed by Tukey's multiple Comparison test), the representative images of transwell chambers coated without (upper panel) or with (lower panel) Matrigel. (C, D) Western blot analysis and the quantification of HEY1, E-cadherin, N-cadherin and Twistl expression in SACC cells after inhibiting HEY1. (E) The mRNA expression of MMP1, MMP2, MMP3, MMP9, MMP11 and MMP13 in SACC cells was detected by real-time PCR when HEY1 was inhibited. $* P<0.05$, $* * P<0.01$, and $* * * \mathrm{P}<0.005$. 


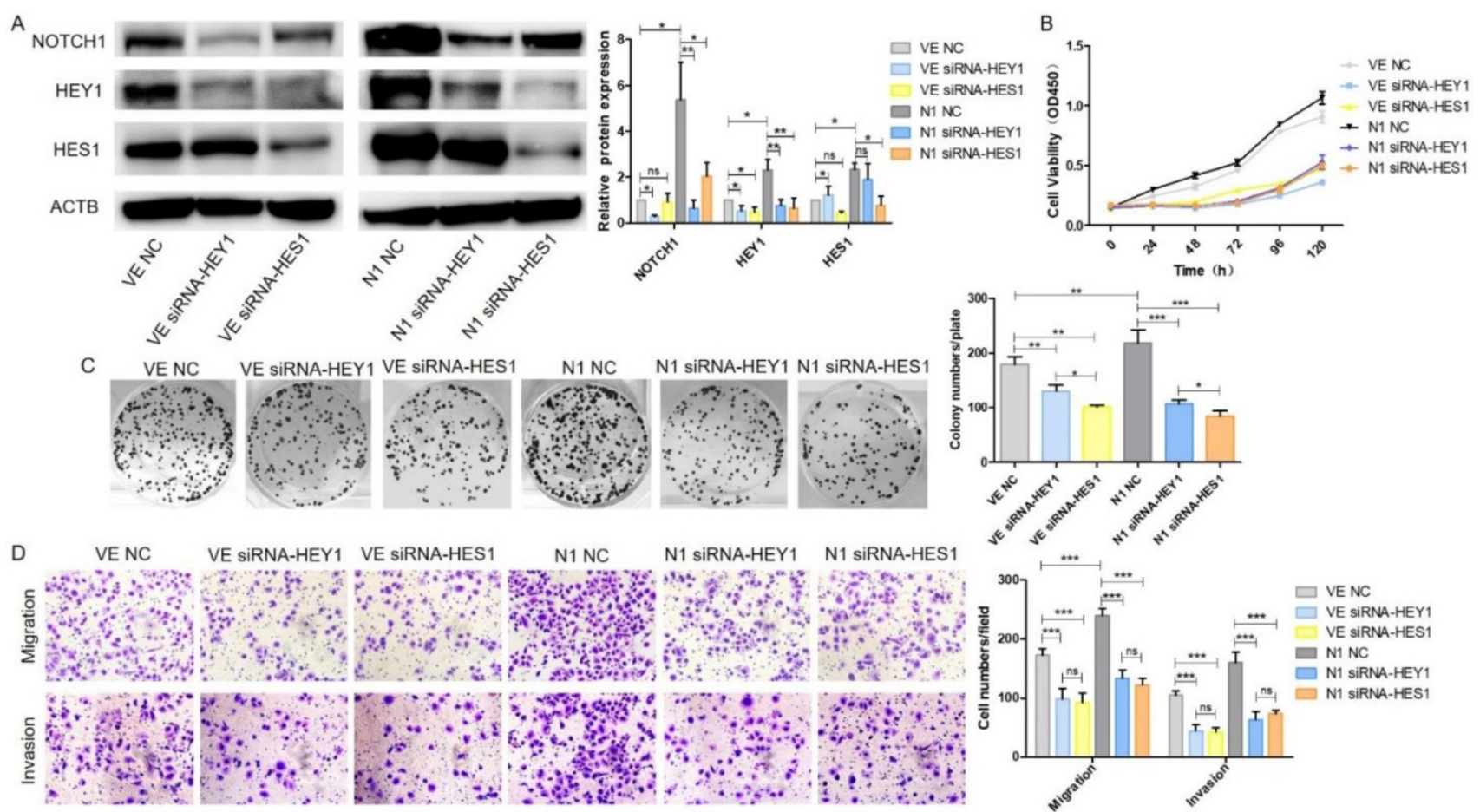

Figure 6. HEYI and HESI contribute equally in mediating NOTCHI signaling pathway in SACC cells. After co-transfection with different plasmid DNA and siRNAs at the same time, the protein expression of NOTCH1, HEY1 and HESI were measured by western blot $(A)$, the cell proliferation was detected by $C C K 8$ assay $(B, P<$ 0.001 by One-Way ANOVA followed by Tukey's multiple Comparison test from $24 \mathrm{~h}$ to $120 \mathrm{~h}$ ) and colony formation assay (C, P<0.05 by One-Way ANOVA followed by Tukey's multiple Comparison test, $n=3$ ). The cell migration and invasion were detected by transwell assay coated with (lower panel) or without (upper panel) matrigel ( $D$, 200x, $\mathrm{P}<0.005$ by One-Way ANOVA followed by Tukey's multiple Comparison test ).

\section{Discussion}

The NOTCH signaling cascade is an evolutionarily highly conserved pathway that plays a crucial role in regulating the growth and development of various tissues and maintaining homeostasis. The abnormal activity of this pathway is also closely related to the occurrence and development of several malignant tumors [25]. Upon the activation of $\mathrm{NOTCH}$ signaling, NOTCH receptors release the signal-transducing $\mathrm{NOTCH}$ intracellular domain (NICD). The NICD migrates into the nucleus and combines with the nuclear proteins of the CSL to form the CSL-NICD transcriptional complex and initially target HEY1 directly [26, 27]. HEY1 plays crucial roles in the development of various tissues and organs and the occurrence and development of tumors [28-30].

In the classical NOTCH signaling pathway, HEY1 is generally activated by NOTCH receptors. Ilaria Saltarella et al found that the knockdown of NOTCH1/2 in multiple myeloma inhibited the expression of the downstream transcription factor HES1/HEY1, which restrained tumor angiogenesis [30]. However, in a study of the association between NOTCH1 and HEY1 in HNSCC, it was shown that HEY1 expression was independent of the activation of NOTCH1, was not associated with either JAG1 or NICD1 expression, and the high expression of HEY1 was associated with a poor prognosis in patients with HNSCC [32]. Takahito Fukusumi and colleagues indicated that $\mathrm{NOTCH} 4$ specifically activates its downstream target gene HEY1 in HNSCC. Conversely, HEY1 can also regulate the expression of $\mathrm{NOTCH} 4$ [17]. In our results, we demonstrated that NOTCH1 overexpression increased the expression of HEY1 in SACC cells, whereas the knockdown of HEY1 by siRNAs significantly decreased the expression of NOTCH1. Furthermore, the expression of NOTCH1 and HEY1 exhibited clearly positive correlation in adenoid cystic carcinoma tissues. Nonetheless, it is still unknown whether HEY1 regulates NOTCH1 expression through direct regulation or another pathway. These results indicate that the NOTCH1-HEY1 pathway could generate a positive feedback loop instead of one-way regulation in SACC.

Wang et al reported that compared with adjacent tissues, HEY1 expression was significantly elevated in Kaposi's sarcoma tissue, and HEY1 promoted tumor angiogenesis [33]. Jung et al. examined the relationship between clinicopathological factors and HEY1 expression in 109 cases of papillary thyroid carcinoma. HEY1 immunoreactivity was positively correlated with lymph node metastasis, recurrence and metastasis [34]. However, Ménard $M$ et al showed that HEY1 inhibited tumor progression by regulating 
tropomyosin receptor kinase $\mathrm{C}$ (TrkC) activity in neuroblastoma [35]. Through the anta gonistic effects of HEY1 on androgen receptor (AR), NOTCH signaling can possibly antagonize malignant prostate growth [29]. HEY1 plays different roles in different tumors. To date, there is no related report on the role of HEY1 in SACC. Our immunohistochemistry results showed that the expression of HEY1 in salivary adenoid cystic carcinoma was significantly higher than that in adjacent tissues. We speculated that HEY1 might promote the occurrence and development of SACC. Brian C. Belyea and colleagues found that abnormal NOTCH-HEY1 signaling promotes tumor progression by blocking cell differentiation and promoting cell proliferation in embryonic subtypes of rhabdomyosarcoma [36]. Thus, we next examined HEY1 functions in SACC and showed that HEY1 enhanced cell proliferation in vitro and tumorigenicity in vivo. In addition, the results of our apoptosis analysis revealed that HEY1 inhibited cell apoptosis. These results suggest that HEY1 promotes the growth of SACC by regulating cell proliferation and apoptosis.

Previous findings have mainly focused on the relationship between HEY1 and the development of the cardiovascular system. Luis Luna-Zurita et al reported that HEY1 affected endocardial formation by regulating the myocardial EMT and the expression of BMP2 in nonchamber myocardium [37]. In cancer, HEY1 has been reported to be essential for the transforming growth factor- $\beta$-dependent EMT, which is frequently observed in advanced carcinogenesis and is related to several cancer-related pathways [28]. Tumor cells transition between epithelial and mesenchymal states in a highly plastic and dynamic manner [38]. The epithelial-mesenchymal transition (EMT) is a reversible process that promotes cell invasion in which cells lose their epithelial characteristics and acquire mesenchymal characteristics, altering the adhesion molecules on the cell surface and allowing them to migrate more efficiently and invade potential mesenchyme [39-41]. In a study of HNSCC, HEY1 promoted cell invasion and migration by inducing the epithelialmesenchymal transition and enhanced stemness [32]. In our research, we also focused on the relationship between HEY1 and the EMT. The results showed that $\mathrm{N}$-cadherin and Twist1 were significantly decreased, while E-cadherin was obviously increased following the inhibition of HEY1 in SACC cells. The EMT is essential for cancer invasion and metastasis [42]. Our in vitro experiments showed that HEY1 promoted the migration and invasion of SACC cells. We believe that HEY1 promotes cell invasion and metastasis by regulating the epithelial-mesenchymal transition in
SACC.

The NOTCH pathway has been reported to modulate the homeostasis of cancer stem cell-like cells (CSCs) in colorectal cancer [43]. Miranda Brun et al found that HEY1 was associated with increased proliferation and stem cell characteristics in GBM cells [44]. The EMT is closely related to CSCs and tumor resistance [45]. The EMT is thought to be induced in a subset of metastatic cancer stem cells (MCSCs), giving the tumor the ability to metastasize throughout the body [46]. After inhibiting the expression of HEY1 in SACC, we found that the volume and number of tumor cell spheroids were significantly reduced. The EMT-induced genes and CSC markers Snail1, Twist1 and Fibronectin 1 were decreased after HEY1 expression was downregulated. In addition, we also examined the expression of OCT4 and ALDH1, known CSC markers, which was also downregulated. Aldehyde dehydrogenase 1 (ALDH1) is a cancer stem cell-like cell (CSC) marker in human cancer that is involved in the regulation of NOTCH1 signaling and regulates the formation of ovarian cancer tumor spheres [38, 47]. In HNSCC, OCT4 is considered a key regulator of CSCs, which targets CSCs and may be of potential value in the treatment of HNSCC [48].

Furthermore, in cancer, abnormally hydrolyzed matrix metalloproteinases cause uncontrolled tumor growth, tissue remodeling, inflammation, tissue invasion and metastasis [49]. Pope JL et al found that claudin-1 regulates Notch signaling through the regulation of MMP-9 and p-ERK signaling to regulate intestinal epithelial homeostasis [50]. Qi Zhang et al reported that MMP2, HEY1 and HES1 together regulate the migration of adipose-derived stem cells and chondrocytes in a coculture system [51]. In tumor studies, MMPs induced tumor invasion and distant metastasis in a variety of tumors, such as breast cancer [52], osteosarcoma [53], endometrial cancer [54], and esophageal squamous cell carcinoma [55]. Therefore, we also studied the correlation between HEY1 and MMPs. Real-time PCR analysis revealed that HEY1 positively regulated the expression of MMP1, MMP2, MMP3, MMP9, MMP11 and MMP13. However, it is still unknown how HEY1 affects the expression of MMPs. Zhang et al found that unbalanced expression of MMP/TIMP axis genes in tumors was corrected with a simple defined factor-mediated reprogramming for nuclear remodeling to change the morphology of tumor cells and to inhibit the migration and invasion of tumor cells [56]. MMP is widely used as a cancer biomarker and therapeutic target. Recently, MMP-based nanomaterials and carriers have emerged in the tumor-targeted delivery of drugs and as imaging agents at the tissue, cellular, and intracellular levels [57]. These results will also 
provide a new direction for the invasion and metastasis of SACC based on HEY1 and MMPs.

In summary, we demonstrate that the NOTCH1-HEY1 pathway is specifically upregulated in SACC and promotes the EMT and MMP expression. HEY1 regulates cell proliferation, spheroid formation, invasion, metastasis and apoptosis inhibition in vitro and in vivo. Therefore, this finding provides new insight into the role of the $\mathrm{NOTCH}$ signaling pathway in SACC carcinogenesis.

\section{Acknowledgements}

We thank Dr. Guo Chuanbin for the kind gift of SACC-LM and SACC-83 cells. This work was supported by the National Natural Sciences Foundation of China [grant number 81172583, 81572286 and 81641105]; the Natural Sciences Foundation of Fujian [grant numbers 2010J01157 and 2011J01167]; the Key Project of Science and Technology Foundation of Fujian Province of China [grant number 2011Y0025]; Scientific research funding of School and Hospital of Stomatology, Fujian Medical University [grant number 2018KQYJ01]

\section{Competing Interests}

The authors have declared that no competing interest exists.

\section{References}

[1] Conley J and Dingman DL. Adenoid Cystic Carcinoma in the Head and Neck (Cylindroma). Archives of Otolaryngology. 1974; 100: 81.

[2] Spiro RH. Distant metastasis in adenoid cystic carcinoma of salivary origin [J]. American Journal of Surgery. 1997; 174: 495.

[3] Kokemueller H, Eckardt A, Brachvogel P, et al. Adenoid cystic carcinoma of the head and neck--a 20 years experience. Int J Oral Maxillofac Surg. 2004; 33(1):25-31.

[4] Je VDW, Becking AG, Snow GB, et al. Distant metastases of adenoid cystic carcinoma of the salivary glands and the value of diagnostic examinations during follow-up. Head \& Neck. 2002; 24: 779.

[5] Yang WW, Yang LQ, Zhao F, et al. Epiregulin Promotes Lung Metastasis of Salivary Adenoid Cystic Carcinoma. Theranostics. 2017; 7: 3700-3714.

[6] Koch U and Radtke F. Notch signaling in solid tumors. Current Topics in Developmental Biology. 2010; 92: 411.

[7] Gordon WR, Arnett KL and Blacklow SC. The molecular logic of Notch signaling - A structural and biochemical perspective. Journal of Cell Science. 2008; 21: 3109.

[8] Meierstiegen F, Schwanbeck R, Bernoth K, et al. Activated NOTCH1 target genes during embryonic cell differentiation depend on the cellular context and include lineage determinants and inhibitors. Plos One. 2010; 5: e11481.

[9] Davis RL and Turner DL. Vertebrate hairy and Enhancer of split related proteins: transcriptional repressors regulating cellular differentiation and embryonic patterning. Oncogene. 2001; 20: 8342.

[10] Iso T, Kedes L and Hamamori Y. HES and HERP families: multiple effectors of the Notch signaling pathway. J Cell Physiol. 2003; 194: 237-255.

[11] Su BH, Qu J, Song M, Huang XY, et al. NOTCH1 signaling contributes to cell growth, anti-apoptosis and metastasis in salivary adenoid cystic carcinoma. Oncotarget. 2014; 5: 6885-6895.

[12] Iso T, Chung G, Hamamori Y, et al. HERP1 Is a Cell Type-specific Primary Target of Notch. Journal of Biological Chemistry. 2002; 277: 6598

[13] Iso T, Sartorelli V, Poizat C, et al. HERP, a novel heterodimer partner of HES/E(spl) in Notch signaling. Molecular \& Cellular Biology. 2001; 21: 6080-6089.
[14] Steidl C, Leimeister C, Klamt B, et al. Characterization of the human and mouse HEY1, HEY2, and HEYL genes: cloning, mapping, and mutation screening of a new bHLH gene family. Genomics. 2000; 66: 195.

[15] Stifani S, Blaumueller CM, Redhead NJ, et al. Human homologs of a Drosophila Enhancer of split gene product define a novel family of nuclear proteins. Nat Genet. 1992, 2(4):343.

[16] Huang XY, et al. The oncogenic effects of HES1 on salivary adenoid cystic carcinoma cell growth and metastasis. BMC Cancer. 2018; 18(1):436.

[17] Fukusumi T, Guo TW, Sakai A, et al. The NOTCH4-HEY1 Pathway Induces Epithelial-Mesenchymal Transition in Head and Neck Squamous Cell Carcinoma. Clin Cancer Res.2018; 24(3):619-633.

[18] Nandagopal N, Santat LA, LeBon L, et al. Dynamic Ligand Discrimination in the Notch Signaling Pathway. Cell. 2018; 172(4 e19): 869-880.

[19] Luna-Zurita L, Prados B, Grego-Bessa J, et al. Integration of a Notch-dependent mesenchymal gene program and Bmp2-driven cell invasiveness regulates murine cardiac valve formation. J Clin Invest. 2010; 120(10):3493-507.

[20] Luisana Astudillo, Thiago G. Da Silva, Zhiqiang Wang, et al. The Small Molecule IMR-1 Inhibits the Notch Transcriptional Activation Complex to Suppress Tumorigenesis. Cancer Res. 2016; 76(12):3593-603.

[21] Sutherland RM, McCredie JA, Inch WR. Growth of multicell spheroids in tissue culture as a model of nodular carcinomas. J Natl Cancer Inst. 1971; 46(1):113-20.

[22] Fischer A, Steidl C, Wagner TU, et al. Combined loss of Hey1 and HeyL causes congenital heart defects because of impaired epithelial to mesenchymal transition. Circ Res. 2007; 100:856-63.

[23] De Craene B and Berx G. Regulatory networks defining EMT during cancer initiation and progression. Nat Rev Cancer. 2013; 13:97-110.

[24] Pastushenko I, Brisebarre A, Sifrim A, et al. Identification of the tumour transition states occurring during EMT. Nature. 2018; 556(7702):463-468.

[25] Nowell CS, Radtke F. Notch as a tumour suppressor. Nat Rev Cancer. 2017; 17(3):145-159.

[26] Maier MM and Gessler M. Comparative analysis of the human and mouse Hey1 promoter: Hey genes are new Notch target genes. Biochemical \& Biophysical Research Communications. 2000; 275: 652.

[27] Iso T, Sartorelli V, Chung G, et al. HERP, a New Primary Target of Notch Regulated by Ligand Binding[J]. Molecular \& Cellular Biology. 2001; 21: 6071

[28] Massari ME and Murre C. Helix-loop-helix proteins: regulators of transcription in eucaryotic organisms. Mol Cell Biol. 2000; 20: 429-440.

[29] Carvalho FL, Simons BW, Eberhart CG, et al. Notch signaling in prostate cancer: a moving target. Prostate. 2014; 74: 933-945.

[30] Lau EY, Lo J, Cheng BY, et al. Cancer-Associated Fibroblasts Regulate Tumor-Initiating Cell Plasticity in Hepatocellular Carcinoma through c-Met/FRA1/HEY1 Signaling. Cell Rep. 2016; 15(6):1175-89.

[31] Saltarella I, et al. Homotypic and Heterotypic Activation of the Notch Pathway in Multiple Myeloma-Enhanced Angiogenesis: A Novel Therapeutic Target. Neoplasia. 2019; 21(1): 93-105.

[32] Rettig EM, Bishop JA, Agrawal N, et al. HEY1 is expressed independent of NOTCH1 and is associated with poor prognosis in head and neck squamous cell carcinoma. Oral Oncol. 2018; 82:168-175.

[33] Wang X, He Z, Xia T, et al. Latency-associated nuclear antigen of Kaposi sarcoma-associated herpesvirus promotes angiogenesis through targeting notch signaling effector Hey1. Cancer Res. 2014; 74(7):2026-37.

[34] Jung CW, Kong JS, Seol H, et al. Expression of activated NOTCH1 and HEY1 in papillary thyroid carcinoma. Histopathology. 2017; 70(2):301-308

[35] Ménard M, Costechareyre C, Ichim G, et al. Hey1- and p53-dependent TrkC proapoptotic activity controls neuroblastoma growth. PLoS Biol. 2018;16(5):e2002912.

[36] Belyea BC, Naini S, Bentley RC, et al. Inhibition of the Notch-Hey1 axis blocks embryonal rhabdomyosarcoma tumorigenesis. Clin Cancer Res. 2011; 17(23):7324-36.

[37] Luna-Zurita L, Prados B, Grego-Bessa J, et al. Integration of a Notch-dependent mesenchymal gene program and Bmp2-driven cell invasiveness regulates murine cardiac valve formation. J Clin Invest. 2010; 120:3493-507.

[38] Nieto MA, Huang RY, Jackson RA, et al. EMT: 2016. Cell. 2016; 166(1):21-45.

[39] Pastushenko I, Brisebarre A, Sifrim A, et al. Identification of the tumour transition states occurring during EMT. Nature. 2018; 556(7702):463-468.

[40] Gupta S, Maitra A. EMT: Matter of Life or Death? Cell. 2016; 164(5):840-2.

[41] Nieto MA, Huang RY, Jackson RA, et al. EMT: 2016. Cell. 2016; 166(1):21-45.

[42] Peng JM, Bera R, Chiou CY, et al. Actin cytoskeleton remodeling drives epithelial-mesenchymal transition for hepatoma invasion and metastasis in mice. Hepatology. 2018; 67(6):2226-2243. 
[43] Jin L, Vu T, Yuan G, et al. STRAP Promotes Stemness of Human Colorectal Cancer via Epigenetic Regulation of the NOTCH Pathway. Cancer Res. 2017; 77(20):5464-5478.

[44] Brun M, Jain S, Monckton EA, et al. Nuclear Factor I Represses the Notch Effector HEY1 in Glioblastoma. Neoplasia. 2018; 20(10) :1023-1037.

[45] Shibue T, Weinberg RA. EMT, CSCs, and drug resistance: the mechanistic link and clinical implications. Nat Rev Clin Oncol. 2017; 14(10):611-629.

[46] Singh M, Yelle N, Venugopal C, et al. EMT: Mechanisms and therapeutic implications. Pharmacol Ther. 2018; 182:80-94.

[47] Young MJ, Wu YH, Chiu WT, et al. All-trans retinoic acid downregulates ALDH1-mediated stemness and inhibits tumour formation in ovarian cancer cells. Carcinogenesis. 2015; 36(4):498-507

[48] Koo BS, Lee SH, Kim JM, et al. Oct4 is a critical regulator of stemness in head and neck squamous carcinoma cells. Oncogene. 2015; 34(18):2317-2324.

[49] Kessenbrock K, Plaks V, Werb Z. Matrix metalloproteinases: regulators of the tumor microenvironment. Cell. 2010; 141(1):52-67.

[50] Pope JL, Bhat AA, Sharma A, et al. Claudin-1 regulates intestinal epithelial homeostasis through the modulation of Notch-signalling. Gut. 2014; 63(4):622-634.

[51] Zhang Q, Deng S, Sun K, et al. MMP-2 and Notch signal pathway regulate migration of adipose-derived stem cells and chondrocytes in co-culture systems. Cell Prolif. 2017; 50(6).

[52] Wu Z, Wang T, Fang M, et al. MFAP5 promotes tumor progression and bone metastasis by regulating ERK/MMP signaling pathways in breast cancer. Biochem Biophys Res Commun. 2018; 498(3):495-501.

[53] Waresijiang N, Sun J, Abuduaini R, et al. The downregulation of miR-125a-5p functions as a tumor suppressor by directly targeting MMP-11 in osteosarcoma. Mol Med Rep. 2016; 13(6):4859-4864.

[54] Jedryka M, Chrobak A, Chelmonska-Soyta A, et al. Matrix metalloproteinase (MMP)-2 and MMP-9 expression in tumor infiltrating CD3 lymphocytes from women with endometrial cancer. Int J Gynecol Cancer. 2012; 22(8):1303-9.

[55] Yang L, Song $X$, Zhu J, et al. Tumor suppressor microRNA-34a inhibits cell migration and invasion by targeting MMP-2/MMP-9/FNDC3B in esophageal squamous cell carcinoma. Int J Oncol. 2017; 51(1):378-388.

[56] Zhang S, Zhong B, Chen M, et al. Epigenetic reprogramming reverses the malignant epigenotype of the MMP/TIMP axis genes in tumor cells. Int J Cancer. 2014; 134(7):1583-94.

[57] Yao Q, Kou L, Tu Y, et al. MMP-Responsive 'Smart' Drug Delivery and Tumor Targeting. Trends Pharmacol Sci. 2018; 39(8):766-781. 